\title{
Predictors of Toxoplasma gondii IgG Seropositivity and Cranial Ultrasound Patterns among Children with Hydrocephalus
}

\author{
Sofia Ottaru, ${ }^{1}$ Mariam M. Mirambo, ${ }^{2}$ Rogatus Kabyemera, ${ }^{1}$ Benson R. Kidenya, ${ }^{3}$ \\ Mwanaisha Seugendo, ${ }^{1}$ Delfina R. Msanga ${ }^{D},{ }^{1}$ Patrick Ngoya, ${ }^{4}$ Domenica Morona $\mathbb{D}^{5}{ }^{5}$ \\ and Stephen E. Mshana ${ }^{2}{ }^{2}$ \\ ${ }^{1}$ Department of Paediatrics \& Child Health, Bugando Medical Centre, P.O. Box 370, Mwanza, Tanzania \\ ${ }^{2}$ Department of Microbiology and Immunology, Weill Bugando School of Medicine, Catholic University of Health and Allied Sciences, \\ P.O. Box 1464, Mwanza, Tanzania \\ ${ }^{3}$ Department of Biochemistry and Molecular Biology, Weill Bugando School of Medicine, Catholic University of Health and \\ Allied Sciences, P.O. Box 1464, Mwanza, Tanzania \\ ${ }^{4}$ Department of Radiology, Bugando Medical Centre, P.O. Box 370, Mwanza, Tanzania \\ ${ }^{5}$ Department of Medical Parasitology and Entomology, Weill Bugando School of Medicine, Catholic University of Health and \\ Allied Sciences, P.O. Box 1464, Mwanza, Tanzania
}

Correspondence should be addressed to Domenica Morona; dmorona@gmail.com

Received 9 June 2020; Revised 27 July 2020; Accepted 28 August 2020; Published 7 September 2020

Academic Editor: Francesco Porta

Copyright (C) 2020 Sofia Ottaru et al. This is an open access article distributed under the Creative Commons Attribution License, which permits unrestricted use, distribution, and reproduction in any medium, provided the original work is properly cited.

Background. Toxoplasma gondii infection during pregnancy is associated with serious neonatal complications, including hydrocephalus. In many high-income countries, T. gondii screening and treatment during the antenatal period are routinely carried out to prevent associated complications, whereas in most low-income countries, there is no routine screening of $T$. gondii during pregnancy. Despite the parasite being common in Tanzania, there is a paucity of information on the prevalence of T. gondii and cranial ultrasound patterns among children with hydrocephalus. Methods. An analytical cross-sectional hospitalbased study involving 125 infants with hydrocephalus attending the Bugando Medical Centre (BMC) was conducted between May 2017 and February 2018. Sociodemographic and other relevant information was collected using a pretested data collection tool. Venous blood samples were collected, and sera were used for the detection of specific T. gondii antibodies by indirect enzyme-linked immunosorbent assay (ELISA) as per manufacturer's instructions. Data were analysed using STATA version 13 software. Results. The mean age of enrolled children was $4.8 \pm 3.5$ months. Out of 125 infants with hydrocephalus, 29 (23.2\%, 95\% CI: 21-36) were seropositive for T. gondii-specific IgG antibodies. By multiple generalized linear model analysis, being male $(\mathrm{aRR}=1.1,95 \%$ CI: $0.9-1.5, p=0.049)$, higher birth order $(\mathrm{aRR}=1.2,95 \%$ CI: $1.0-1.5, p=0.023)$, consumption of fish meat $(\mathrm{aRR}=1.6,95 \% \mathrm{CI}: 1.2-2.3, p=0.003)$, and using other methods of cooking meat than boiling $(\mathrm{aRR}=1.7,95 \% \mathrm{CI}: 1.1-2.5, p=$ 0.015) were independent risk factors for T. gondii IgG seropositivity. Obstructive hydrocephalus was significantly more common among T. gondii-seronegative infants compared to IgG-seropositive infants (31.3\% [30/96] vs. 13.8\% [4/29]; $p=0.049)$. Conclusions. A significant proportion of infants with nonobstructive hydrocephalus are T. gondii IgG seropositive, and this is predicted by male gender, increase of birth order, consuming fish, and using other methods of cooking meat than boiling. These facts highlight the importance of continuing health education for pregnant women regarding T. gondii transmission and the need to follow-up their infants so that appropriate counselling and management can be provided.

\section{Background}

Toxoplasma gondii infection is a public health problem with detrimental effect to the developing fetus. The mother-to- child transmission risk of $T$. gondii infection is estimated to be $29 \%$, and seropositivity has been found to increase with an increase in age and gestation age [1-4]. Acquisition of this infection during pregnancy, especially in the first trimester, 
can lead to serious neonatal complications which include retinochoroiditis, hydrocephalus, intracranial calcifications, and convulsions [5].

Hydrocephalus (HDC) is a disturbance of the cerebrospinal fluid (CSF) formation, flow, or absorption, leading to a high volume of CSF in the central nervous system (CNS) [6]. About 750,000 children and adults are living with hydrocephalus, and one in every 500 live births is affected worldwide [7]. In high-income countries (HIC), the incidence of congenital hydrocephalus has been estimated at 0.5 cases per 1000 live births and the incidence of neonatal hydrocephalus has been estimated at 3-5 cases per 1000 live births [8]. In the East African region, more than 6000 new cases of hydrocephalus occur every year [9].

Congenital toxoplasmosis (CT) has been found to be common particularly when infection occurs in the first trimester; a previous study reported that about $67.7 \%$ of children with CT had hydrocephalus, indicating that $T$. gondii is one of the common contributing factors to these cases [10]. The review of the surgical ward admission book at the Bugando Medical Centre (BMC) in 2016 showed that hydrocephalus was the leading cause of admission in children, with at least 15 to 20 new cases admitted every month. Moreover, a previous study by Mashuda et al. reported that $35.9 \%$ of the children with congenital malformations had hydrocephalus, making it the second most occurring CNS congenital malformation among infants at BMC [11]. Additionally, previous studies in Mwanza and Dar es Salaam, Tanzania, reported a $T$. gondii IgG seropositivity of about $31 \%$ among pregnant women and $55.4 \%$ among women with spontaneous abortions $[4,12,13]$; this indicates an increased risk of congenital $T$. gondii infection among unborn children in these settings. Despite a high occurrence of hydrocephalus and a high seroprevalence of $T$. gondii among pregnant women in Mwanza, the association between $T$. gondii seropositivity and hydrocephalus has not been studied in this setting. Likewise, cranial ultrasound patterns among infants with hydrocephalus are not well understood in many centres in developing countries. As a matter of fact, control interventions require the formulation of an evidence-based policy to set guidelines for screening and treating pregnant women in countries where T. gondii is endemic. This study is therefore aimed at determining the seropositivity of $T$. gondii antibodies and its predictors and cranial ultrasound patterns among infants with hydrocephalus.

\section{Methodology}

2.1. Study Design and Duration. An analytical cross-sectional hospital-based study involving 125 infants aged 0-12 months with hydrocephalus was conducted from September 2017 to April 2018.

2.2. Study Site. The study was conducted at the departments of paediatrics and child health and surgery (neurology) of BMC, a referral and teaching hospital for the Lake Victoria and Western Zones of the United Republic of Tanzania. This centre for tertiary specialist care serves eight regions, including Mwanza, Geita, Simiyu, Mara, Kagera, Shinyanga, Tabora, and Kigoma, with a catchment population of approximately 14 million people [14]. The department of paediatrics and child health has a capacity of 121 beds. It is subdivided into general paediatric wards, malnutrition wards, semi-intensive care unit, neonatal unit, neonatal intensive care unit, and outpatient department. Approximately twenty new cases of hydrocephalus are admitted in the surgical departments every month, while in paediatric wards approximately five new cases are admitted every month.

2.3. Study Population and Selection Criteria. The study included all infants with hydrocephalus who attended the surgical and paediatric departments of Bugando Medical Centre during the study period. Infants who were critically ill or did not have brain ultrasounds or CT scan results were excluded.

2.4. Sample Size and Sampling Procedure. For sample size calculation, a formula for comparing two independent sample sizes was used. We assumed the proportion of infants who will be IgG positive to be $8 \%$ and those who will be IgM positive to be $0.115 \%$, at $95 \%$ confidence interval [15]; the minimum sample size was 125 infants. In a period of 7 months, a total of 290 children with hydrocephalus were attended at BMC, 62 children from the paediatric department and 228 from the surgery department-neurology clinic. A total of 144 children who were above 1 year were excluded from the study. Out of 146 infants who were eligible for the study, 125 (85.6\%) infants were conveniently enrolled (Figure 1).

2.5. Data Collection Procedure. Detailed history taking and thorough physical examination were performed for all the children enrolled. The information collected included sociodemographic characteristics, maternal details (residency, level of education), and risk factors (like owning a cat, eating undercooked meat, gardening, and drinking unboiled water) associated with $T$. gondii infections. The HIV status of the mother and child's risk factors (like crawling and eating soil) were also noted.

2.6. Sample Collection and Laboratory Procedures. Three millilitres $(3 \mathrm{ml})$ of blood was collected from each participant and placed in plain vacutainer tubes (Neomedic Limited, China) for the detection of T. gondii-specific IgM and IgG antibodies by using the enzyme-linked immunosorbent assay (ELISA). The blood samples were taken to CUHAS-Bugando multipurpose laboratory where sera were separated from the whole blood by centrifugation at $3000 \mathrm{rpm}$ for 5 minutes. Sera were kept at $-40^{\circ} \mathrm{C}$ until processing. Specific T. gondii IgM and IgG antibodies were detected by indirect ELISA (Pishtaz Teb Diagnostics, Tehran, Iran) [16], with sensitivity and specificity for IgM of $100 \%$ and $99 \%$, respectively, and sensitivity and specificity for IgG of $100 \%$. All procedures were performed following the manufacturer's instructions and the standard operating procedures of the BMC accredited laboratory.

2.7. Cranial Ultrasound Scan. For each study participant, the occipital frontal circumference (OFC) was measured followed by cranial ultrasounds or head CT scan (as an 


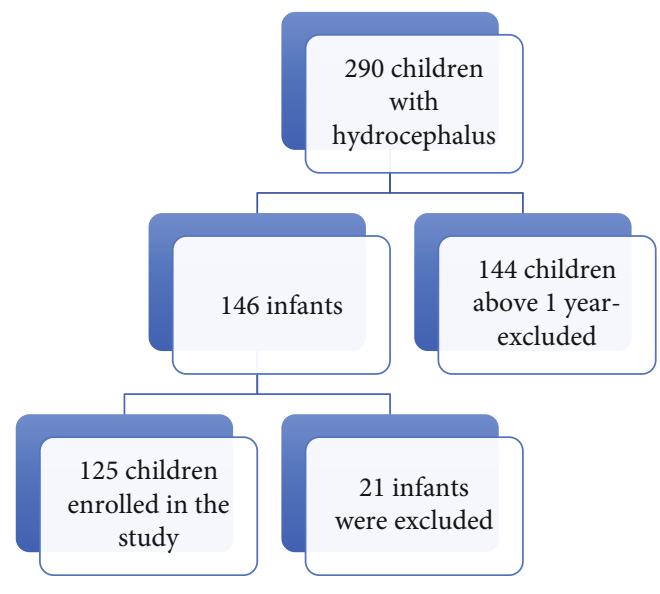

FIGURE 1: Study enrolment.

alternative mode of confirmation) to confirm the diagnosis of hydrocephalus. A cranial ultrasound scan was performed, and anatomical patterns of hydrocephalus were denoted as follows: obstruction of the aqueduct of Sylvius, bilateral obstruction of the foramina of Monro, unilateral obstruction of the foramina of Monro, and no obvious intraventricular obstruction causing ventricular dilatation [17]. In addition, any other structural anomalies were documented.

2.8. Data Quality Control. Before analysing the blood samples for $T$. gondii seropositivity, the test kits were calibrated by using the provided standards and controls. In addition, for each test run, positive and negative controls were included. Results were only considered valid if the controls and standard passed the test.

2.9. Data Management and Analysis. Data collected were recorded into a computer using Microsoft Office Excel 2007. Data was analysed with the STATA version 12 software (College Station, Texas, USA). Categorical variables were summarized as percentages and analysed by chi-square or Fisher's exact tests where appropriate. Continuous variables were summarized as mean (standard deviation) or median (interquartile range) where appropriate. Univariate and multivariate analyses were performed using a generalized linear model to determine the risk factors of $T$. gondii seropositivity. Risk factors with a $p$ value less than 0.20 on univariate analysis were fitted into the multivariate analysis; risk ratios and $95 \%$ confidence intervals were noted. Risk factors with $p$ values less than 0.05 were considered statistically significant.

\section{Results}

3.1. Sociodemographic Characteristics. The mean age of the enrolled children was $4.8 \pm 3.5$ months. Forty-eight (38.4\%) were aged 2-4 months, and most of them 76/125 (60.8\%) were male. Most of the infants 76/125 (60.8\%) were from rural areas of the city, while in birth order 53/125 (42.4\%) they lay between being second and fourth born (Table 1).
TABlE 1: Sociodemographic characteristics and other relevant characteristics among infants and their mothers.

\begin{tabular}{|c|c|c|}
\hline Children characteristics & Number $(n)$ & Percentage (\%) \\
\hline \multicolumn{3}{|l|}{ Age group } \\
\hline Neonates & 30 & 24.0 \\
\hline Early infancy & 48 & 38.4 \\
\hline Late infancy & 47 & 37.6 \\
\hline \multicolumn{3}{|l|}{ Gender } \\
\hline Female & 49 & 39.2 \\
\hline Male & 76 & 60.8 \\
\hline \multicolumn{3}{|l|}{ Birth order } \\
\hline $1^{\text {st }}$ born & 37 & 29.6 \\
\hline $2^{\text {nd }}-4^{\text {th }}$ born & 53 & 42.4 \\
\hline$>5^{\text {th }}$ born & 35 & 28.0 \\
\hline \multicolumn{3}{|l|}{ Residence } \\
\hline Urban & 49 & 39.2 \\
\hline Rural & 76 & 60.8 \\
\hline \multicolumn{3}{|c|}{ * Caregiver's level of education } \\
\hline Incomplete & 33 & 26.4 \\
\hline Primary school & 70 & 56.0 \\
\hline Secondary school & 22 & 17.6 \\
\hline \multicolumn{3}{|l|}{ * Caregiver's employment } \\
\hline Peasants & 84 & 67.2 \\
\hline Not employed & 18 & 14.4 \\
\hline Employed & 23 & 18.4 \\
\hline \multicolumn{3}{|l|}{ * Marital status } \\
\hline Not married & 16 & 12.8 \\
\hline Married & 109 & 87.2 \\
\hline \multicolumn{3}{|l|}{ * Maternal age } \\
\hline$<35$ years & 115 & 92.0 \\
\hline$>35$ years & 10 & 8.0 \\
\hline
\end{tabular}

* Mothers/guardians' characteristics.

3.2. Clinical Characteristics. Out of 125 enrolled children, the mean occipital frontal circumference was $48.4 \pm 6.8 \mathrm{~cm}$ and $36 / 125$ (28.8\%) infants had a history of neural tube defects. A history of fever and convulsions before the onset of hydrocephalus was observed in 28/125 (22.4\%) and 22/125 (17.6\%) infants, respectively (Table 2).

3.3. Seropositivity of Specific T. gondii Antibodies among Infants with Hydrocephalus. Out of 125 infants with hydrocephalus tested for T. gondii-specific antibodies, 29/125 (23.2\%, 95\% CI: 16.5-31.5) were found to be IgG seropositive while none of them was IgM seropositive.

3.4. Risk Factors Associated with T. gondii-Specific IgG Seropositivity among 125 Infants with Hydrocephalus. On bivariate analysis, a high birth order $(\mathrm{RR}=1.3,95 \% \mathrm{CI}$ : 1.0 $1.5, p=0.004$ ) was significantly associated with specific $T$. gondii IgG seropositivity (Table 3 ). In addition, consuming fish ( $\mathrm{RR}=1.6,95 \% \mathrm{CI}: 1.1-2.2, p=0.008)$, and using different meat preparation methods (roasting, frying, deep frying, etc.) 
TABLE 2: Clinical characteristics of 125 infants with hydrocephalus.

\begin{tabular}{lcc}
\hline Characteristics & Number $(n)$ & Percentage (\%) \\
\hline Neural tube defect & & \\
$\quad$ No & 89 & 71.2 \\
$\quad$ Yes & 36 & 28.8 \\
History of fever before HDC & & \\
$\quad$ No & 97 & 77.6 \\
$\quad$ Yes & 28 & 22.4 \\
History of convulsions before HDC & & \\
No & 103 & 82.4 \\
$\quad$ Yes & 22 & 17.6 \\
Developmental milestone & & \\
$\quad$ Delayed & 60 & 48.0 \\
Regressed & 4 & 3.2 \\
$\quad$ Up to date & 61 & 48.8 \\
\hline
\end{tabular}

other than boiling meat ( $\mathrm{RR}=1.7,95 \% \mathrm{CI}: 1.1-2.6, p=0.011)$ were significantly associated with specific $T$. gondii IgG seropositivity (Table 4). By multiple generalized linear model analysis, male gender $(\mathrm{aRR}=1.1,95 \% \mathrm{CI}: 0.9-1.5, p=0.049)$, consuming fish more than four times in a week $(\mathrm{RR}=1.6$, 95\% CI: $1.2-2.3, \quad p=0.003)$, increasing birth order $(\mathrm{aRR}=1.2,95 \% \mathrm{CI}: 1.0-1.5, p=0.023)$, and using different methods of cooking other than boiling ( $\mathrm{aRR}=1.7,95 \% \mathrm{CI}$ : $1.1-2.5, p=0.015)$ were found to predict specific $T$. gondii IgG seropositivity (Table 5).

3.5. Cranial Ultrasound Patterns. Among 125 infants with hydrocephalus, the majority, 82/125 (65.6\%), had no obvious obstruction while other patterns reported were obstructive (Dandy Walker cyst, Arnold Chiari malformation) (Figure 2). Further analysis showed that IgG seropositivity was likely to occur in infants with no obvious obstruction as compared to others; however, it was not statistically significant (Figure 3). Overall obstructive hydrocephalus was borderline significantly more common among $T$. gondii IgG-seronegative infants than in IgG-seropositive infants $(31.3 \%$ [30/96] vs. $13.8 \%$ [4/29]; $p=0.049$ ) (Figure 4)

Further analysis of 29 infants with hydrocephalus revealed that their median age was 2 (IQR 1-7) months, most of them were from rural areas $17 / 29$ (58.6\%), and 20/29 $(69.0 \%)$ were male. The mean maternal age was $28.4 \pm 7.3$ years, and severe communicating hydrocephalus was present in $15 / 29$ (51.7\%) (Table 6).

\section{Discussion}

To the best of our knowledge, this is the first study to assess the association between the seropositivity of T. gondii antibodies and hydrocephalus among infants in Africa. In the present study, the seropositivity of specific IgG antibodies was $23.2 \%$ among infants with hydrocephalus which is significantly higher than in infants without hydrocephalus, when compared with the United States, Southeast Brazil, and France that reported a seropositivity of $0.01 \%, 0.1 \%$, and $0.3 \%$, respectively [18-20]. The possible explanation could
TABLE 3: Sociodemographic characteristic risk factors associated with T. gondii seropositivity among 125 enrolled infants and their mothers.

\begin{tabular}{|c|c|c|c|c|}
\hline \multirow{2}{*}{ Factors } & \multicolumn{2}{|c|}{$\begin{array}{c}\text { Toxoplasma } \\
\text { seroprevalence }\end{array}$} & \multirow{2}{*}{$\begin{array}{c}\text { Bivariate RR } \\
\text { [95\% CI] }\end{array}$} & \multirow{2}{*}{$p$ value } \\
\hline & $\begin{array}{c}\text { IgG Pos } \\
N(\%)\end{array}$ & $\begin{array}{c}\text { IgG Neg } \\
N(\%)\end{array}$ & & \\
\hline \multicolumn{5}{|l|}{ Age } \\
\hline Neonates & $8(26.7)$ & $22(73.3)$ & & \\
\hline Early infancy & $12(25.0)$ & $36(75)$ & $1.0[0.8-1.2]$ & 0.867 \\
\hline Late infancy & $9(19.2)$ & $38(80.9)$ & $0.9[0.8-1.1]$ & 0.450 \\
\hline \multicolumn{5}{|l|}{ Gender } \\
\hline Female & $9(18.4)$ & $40(81.6)$ & & \\
\hline Male & $20(26.3)$ & $56(73.7)$ & $1.1[0.9-1.3]$ & 0.306 \\
\hline \multicolumn{5}{|l|}{ Order of birth } \\
\hline $2^{\text {nd }}-4^{\text {th }}$ born & $6(11.3)$ & $47(88.7)$ & & \\
\hline $1^{\text {st }}$ born & $10(27.0)$ & $27(73.0)$ & $1.2[1.0-1.3]$ & 0.076 \\
\hline $\begin{array}{l}5^{\text {th }} \text { born and } \\
\text { above }\end{array}$ & $13(37.1)$ & $22(62.9)$ & $1.3[1.0-1.5]$ & 0.004 \\
\hline \multicolumn{5}{|l|}{ Residence } \\
\hline Rural & $17(22.4)$ & $59(77.6)$ & & \\
\hline Urban & $12(24.5)$ & $37(75.5)$ & $1.0[0.9-1.2]$ & 0.785 \\
\hline \multicolumn{5}{|l|}{ * Education } \\
\hline Incomplete & $10(30.3)$ & $23(69.7)$ & & \\
\hline Primary & $13(18.3)$ & $58(81.7)$ & $0.9[0.7-1.1]$ & 0.179 \\
\hline $\begin{array}{l}\text { Secondary } \\
\text { and above }\end{array}$ & $6(28.6)$ & $15(71.4)$ & $0.9[0.8-1.2]$ & 0.884 \\
\hline \multicolumn{5}{|l|}{ * Occupation } \\
\hline Unemployed & $4(22.2)$ & $14(77.8)$ & & \\
\hline Peasants & $18(21.4)$ & $66(78.6)$ & $1.0[0.8-1.2]$ & 0.943 \\
\hline Employed & $7(30.4)$ & $16(69.6)$ & $1.1[0.8-1.4]$ & 0.540 \\
\hline \multicolumn{5}{|l|}{ * Marital status } \\
\hline Not married & $4(25.0)$ & $12(75.0)$ & & \\
\hline Married & $25(22.9)$ & $84(77.06)$ & $1.0[0.8-1.2]$ & 0.856 \\
\hline \multicolumn{5}{|l|}{ * Maternal age } \\
\hline$<35 \mathrm{yrs}$ & $25(21.7)$ & $90(78.3)$ & & \\
\hline$>35 \mathrm{yrs}$ & $4(40.0)$ & $6(60.0)$ & $1.2[0.9-1.6]$ & 0.190 \\
\hline \multicolumn{5}{|l|}{ Maternal (S) } \\
\hline No & $24(26.1)$ & $68(73.9)$ & & \\
\hline Yes & $5(15.2)$ & $28(84.9)$ & $0.9[0.8-1.1]$ & 0.202 \\
\hline \multicolumn{5}{|l|}{ Animal keeping } \\
\hline No & $9(19.2)$ & $38(80.1)$ & & \\
\hline Yes & $20(25.6)$ & $58(74.4)$ & $1.1[0.9-1.2]$ & 0.407 \\
\hline \multicolumn{5}{|c|}{ * Hand washing } \\
\hline No & $3(18.8)$ & $13(81.3)$ & & \\
\hline Yes & $26(23.9)$ & $83(76.2)$ & $1.1[0.8-1.3]$ & 0.654 \\
\hline \multicolumn{5}{|l|}{ Type of latrines } \\
\hline Latrines & $19(23.8)$ & $61(76.3)$ & & \\
\hline Bush/dustbin & $10(22.2)$ & $35(77.8)$ & $1.0[0.8-1.2]$ & 0.847 \\
\hline
\end{tabular}

* Mothers/guardians' characteristics. 
TABLE 4: Other risk factors associated with T. gondii transmission.

\begin{tabular}{|c|c|c|c|c|}
\hline \multirow{2}{*}{ Factors } & \multicolumn{2}{|c|}{$\begin{array}{c}\text { Toxoplasma } \\
\text { seroprevalence }\end{array}$} & \multirow{2}{*}{$\begin{array}{c}\text { Bivariate RR } \\
{[95 \% \mathrm{CI}]}\end{array}$} & \multirow{2}{*}{$p$ value } \\
\hline & $\begin{array}{l}\text { IgG Pos } \\
N(\%)\end{array}$ & $\begin{array}{c}\text { IgG Neg } \\
N(\%)\end{array}$ & & \\
\hline \multicolumn{5}{|l|}{ Contact with cats } \\
\hline No & $8(18.6)$ & $35(81.4)$ & & \\
\hline Yes & $21(25.6)$ & $61(74.4)$ & $1.0[0.9-1.3]$ & 0.380 \\
\hline \multicolumn{5}{|c|}{ Type of meat consumed } \\
\hline \multicolumn{5}{|c|}{ Fish } \\
\hline No & $25(21.0)$ & $94(79.0)$ & & \\
\hline Yes & $4(66.7)$ & $2(33.3)$ & $1.6[1.1-2.2]$ & 0.008 \\
\hline \multicolumn{5}{|l|}{ Beef } \\
\hline No & $8(24.2)$ & $25(75.8)$ & & \\
\hline Yes & $21(22.8)$ & $71(77.2)$ & $1.0[0.8-1.2]$ & 0.870 \\
\hline \multicolumn{5}{|l|}{ Goat } \\
\hline No & $22(21.6)$ & $80(78.4)$ & & \\
\hline Yes & $7(30.4)$ & $16(69.6)$ & $1.1[0.9-1.3]$ & 0.365 \\
\hline \multicolumn{5}{|l|}{ Pork } \\
\hline No & $27(27.3)$ & $94(77.7)$ & & \\
\hline Yes & $2(50.0)$ & $2(50.0)$ & $1.3[0.9-2.0]$ & 0.197 \\
\hline \multicolumn{5}{|l|}{ Sheep } \\
\hline No & $27(22.3)$ & $94(77.7)$ & & \\
\hline Yes & $2(50.0)$ & $2(50.0)$ & $1.3[0.9-2.0]$ & 0.197 \\
\hline \multicolumn{5}{|l|}{ Meat preparation } \\
\hline Boiling & $26(21.5)$ & $95(78.5)$ & & \\
\hline $\begin{array}{l}\text { Other } \\
\text { methods }\end{array}$ & $3(75.0)$ & $1(25.0)$ & $1.7[1.1-2.6]$ & 0.011 \\
\hline \multicolumn{5}{|l|}{ Grill meat } \\
\hline Never & $20(26.0)$ & $57(74.03)$ & & \\
\hline Everyday/weekly & $6(20.7)$ & $23(79.3)$ & $0.9[0.8-1.1]$ & 0.569 \\
\hline Monthly & $3(15.8)$ & $16(84.2)$ & $0.9[0.7-1.1]$ & 0.350 \\
\hline \multicolumn{5}{|c|}{ Undercooked vegetable } \\
\hline No & $14(20.9)$ & $53(79.1)$ & & \\
\hline Yes & $15(25.9)$ & $43(74.1)$ & $1.1[0.9-1.2]$ & 0.514 \\
\hline \multicolumn{5}{|l|}{ Boiling water } \\
\hline No & $15(19.7)$ & $61(80.3)$ & & \\
\hline Yes & $14(28.6)$ & $35(71.4)$ & $1.1[0.9-1.3]$ & 0.255 \\
\hline \multicolumn{5}{|l|}{ Gardening } \\
\hline No & $16(22.2)$ & $56(77.8)$ & & \\
\hline Yes & $13(24.5)$ & $40(75.5)$ & $1.0[0.9-1.2]$ & 0.765 \\
\hline \multicolumn{5}{|l|}{ Inedible foods } \\
\hline No & $27(22.9)$ & $91(77.1)$ & & \\
\hline Yes & $2(28.6)$ & $5(71.4)$ & $1.0[0.8-1.5]$ & 0.731 \\
\hline \multicolumn{5}{|l|}{ Crawling } \\
\hline No & $27(22.7)$ & $92(77.3)$ & & \\
\hline Yes & $2(33.3)$ & $4(66.7)$ & $1.1[0.8-1.6]$ & 0.549 \\
\hline \multicolumn{5}{|c|}{ Sibling with hydrocephalus } \\
\hline No & $28(22.6)$ & $96(77.4)$ & & \\
\hline Yes & $1(100)$ & $0(0)$ & $2.1[0.9-5.0]$ & 0.066 \\
\hline
\end{tabular}

be the endemicity of T. gondii in the study area whereby IgG seropositivity of $30.9 \%$ has been previously reported among pregnant women in the same setting $[4,13]$. On the other hand, warm climatic conditions in the study area could explain the fact; a warm climate has been found to favour sporulation of oocysts elsewhere [21, 22].

In the current study, none of the infants was found to be IgM seropositive which is consistent with other studies where IgM detection was less than $0.01 \%$ [23]. This could be explained by the possibility that the infants in this study might have seroconverted while in the intrauterine environment, especially before the third trimester. Other possibilities for reactive IgG while $\operatorname{IgM}$ is nonreactive could be if the infection was acquired early in pregnancy, if maternal IgG is able to cross the placenta and suppress the IgM response in the fetus, or if the child was born prematurely with an immature immune system [24]. Moreover, in the current study, T. gondii IgG seropositivity was observed more frequently in neonates than in other age groups, even though the difference was not statistically significant. It has been established that the seropositivity of $T$. gondii tends to increase with an increase in age [25]. There are possibilities that most of the infants in the current study acquired these antibodies from their mother.

In the present study, the risk of being IgG seropositive was higher among male infants than among their female counterparts. However, it is generally documented that no gender predilection occurs with T. gondii seropositivity [26]. Further studies to explore the possibility of increased male gender susceptibility to T. gondii infection are warranted.

In this study, the risk of being IgG seropositive was higher among infants who were fifth born and above compared to their counterparts. This could be explained by the fact that an increase in the birth order is related with an increase in maternal age [27]. In the previous study by Mwambe et al., the risk of being T. gondii seropositive increased by $7 \%$ with a year increase in age [4]. Therefore, these infants were more likely to be born from mothers who were seropositive. This is further supported by the current study whereby mothers with fifth-born children and above were significantly older than those with a firstborn child.

In the current study, consuming fish more than four times a week presented a significantly increased risk of being T. gondii IgG seropositive. This could be explained by the fact that fish is a staple food in Mwanza areas and is usually medium cooked (undercooked). There are limited studies that have investigated $T$. gondii in fish; therefore, more research is needed in this area $[28,29]$. However, due to the small sample size, small number of participants reported to mainly consume fish, pork, and sheep and use other methods to prepare meat; therefore, these results should be interpreted considering this limitation. Outbreaks of toxoplasmosis associated with raw meat consumption have been reported elsewhere [30]. Studies have documented that different animals' meat such as sheep, cattle, beef, duck, pork, and goat could be common sources of T. gondii infection [30-32]. Other works have also reported that $T$. gondii is a common pathogen in fish and its presence in these animals may 
TABLE 5: Risk factors associated with T. gondii seropositivity among infants with hydrocephalus and their mothers.

\begin{tabular}{|c|c|c|c|c|c|c|}
\hline \multirow{2}{*}{ Factors } & \multicolumn{2}{|c|}{ Toxoplasma seroprevalence } & \multirow{2}{*}{ Bivariate RR [95\% CI] } & \multirow{2}{*}{$p$ value } & \multirow{2}{*}{ aRR $[95 \% \mathrm{CI}]$} & \multirow{2}{*}{$p$ value } \\
\hline & IgG Neg $N(\%)$ & IgG Pos $N(\%)$ & & & & \\
\hline \multicolumn{7}{|l|}{ Age } \\
\hline Neonates & $22(73.3)$ & $8(26.7)$ & & & & \\
\hline Early infancy & $36(75.0)$ & $12(25.0)$ & $1.0[0.8-1.2]$ & 0.867 & $1.0[0.8-1.2]$ & 0.912 \\
\hline Late infancy & $38(80.9)$ & $9(19.2)$ & $0.9[0.8-1.1]$ & 0.450 & $0.9[0.7-1.0]$ & 0.133 \\
\hline \multicolumn{7}{|l|}{ Gender } \\
\hline Female & $40(81.6)$ & $9(18.4)$ & & & & \\
\hline Male & $56(73.7)$ & $20(26.3)$ & $1.1[0.9-1.3]$ & 0.306 & $1.1[0.9-1.5]$ & 0.049 \\
\hline \multicolumn{7}{|l|}{ * Education } \\
\hline Incomplete & $23(69.7)$ & $10(30.3)$ & & & & \\
\hline Primary & $58(81.7)$ & $13(18.3)$ & $0.9[0.7-1.1]$ & 0.179 & $0.9[0.7-1.1]$ & 0.211 \\
\hline Secondary and above & $15(71.4)$ & $6(28.6)$ & $0.9[0.8-1.2]$ & 0.884 & $0.9[0.8-1.2]$ & 0.648 \\
\hline \multicolumn{7}{|l|}{ * Maternal age } \\
\hline$<35$ yrs & $90(78.3)$ & $25(21.7)$ & & & & \\
\hline$>35$ yrs & $6(60.0)$ & $4(40.0)$ & $1.2[0.9-1.6]$ & 0.190 & $1.1[0.9-1.5]$ & 0.391 \\
\hline \multicolumn{7}{|l|}{ Order of birth } \\
\hline $2^{\text {nd }}-4^{\text {th }}$ born & $47(88.7)$ & $6(11.3)$ & & & & \\
\hline $1^{\text {st }}$ born & $27(73.0)$ & $10(27.0)$ & $1.2[1.0-1.3]$ & 0.076 & $1.2[1.0-1.4]$ & 0.038 \\
\hline $5^{\text {th }}$ born and above & $22(62.9)$ & $13(37.1)$ & $1.3[1.0-1.5]$ & 0.004 & $1.2[1.0-1.5]$ & 0.023 \\
\hline \multicolumn{7}{|l|}{ Types of meat consumed } \\
\hline \multicolumn{7}{|l|}{ Fish } \\
\hline No & $94(79.0)$ & $25(21.0)$ & & & & \\
\hline Yes & $2(33.3)$ & $4(66.7)$ & $1.6[1.1-2.2]$ & 0.008 & $1.6[1.2-2.3]$ & 0.003 \\
\hline \multicolumn{7}{|l|}{ Pork } \\
\hline No & $94(77.7)$ & $27(27.3)$ & & & & \\
\hline Yes & $2(50.0)$ & $2(50.0)$ & $1.3[0.9-2.0]$ & 0.197 & $1.1[0.7-1.6]$ & 0.801 \\
\hline \multicolumn{7}{|l|}{ Sheep } \\
\hline No & $94(77.7)$ & $27(22.3)$ & & & & \\
\hline Yes & $2(50.0)$ & $2(50.0)$ & $1.3[0.9-2.0]$ & 0.197 & $1.2[0.8-2.0]$ & 0.355 \\
\hline \multicolumn{7}{|l|}{ Meat preparation } \\
\hline Boiling & $95(78.5)$ & $26(21.5)$ & & & & \\
\hline Other methods & $1(25.0)$ & $3(75.0)$ & $1.7[1.1-2.6]$ & 0.011 & $1.7[1.1-2.5]$ & 0.015 \\
\hline \multicolumn{7}{|l|}{ * Maternal (S) } \\
\hline No & $68(73.9)$ & $24(26.1)$ & & & & \\
\hline Yes & $28(84.9)$ & $5(15.2)$ & $0.9[0.8-1.1]$ & 0.202 & $0.8[0.7-1.0]$ & 0.037 \\
\hline \multicolumn{7}{|l|}{ Sibling with hydrocephalus } \\
\hline No & $96(77.4)$ & $28(22.6)$ & & & & \\
\hline Yes & $0(0)$ & $1(100)$ & $2.1[0.9-5.0]$ & 0.066 & $1.5[0.6-3.7]$ & 0.390 \\
\hline
\end{tabular}

$\mathrm{aRR}=$ adjusted risk ratio. $*$ Mothers/guardians' characteristics.

indicate the contamination of the aquatic environment by oocysts of the parasite $[33,34]$. Further studies to establish relationship between toxoplasmosis and fish consumption around Lake Victoria zone are warranted.

In this study, infants whose mothers reported to prefer other methods of meat preparation than boiling, like frying and grilling, had significantly higher odds of being T. gondii IgG seropositive than those who preferred the boiling method. Consuming undercooked meat has been reported as a potential source of $T$. gondii infection as confirmed in the current study [35]. A previous study showed that a temperature of more than $70^{\circ} \mathrm{C}$ is required for the cyst to be destroyed which is not likely to be attained by methods like partial grilling in local settings [36].

Our results show that infants whose mothers reported symptoms suggestive of $T$. gondii infection during pregnancy were less likely to be $T$. gondii seropositive. This could be explained by the fact that the presence of these symptoms 


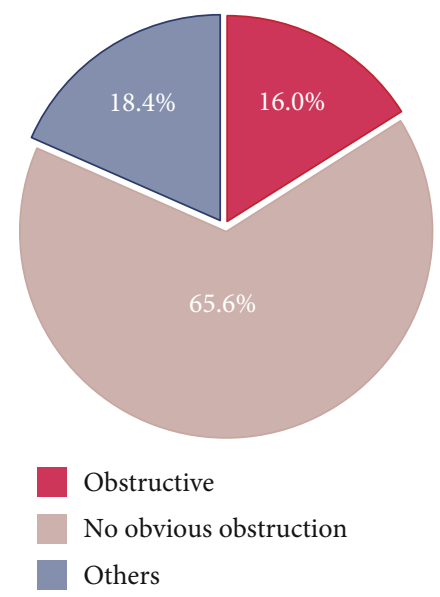

Figure 2: Overview of cranial ultrasound patterns in 125 infants with hydrocephalus.

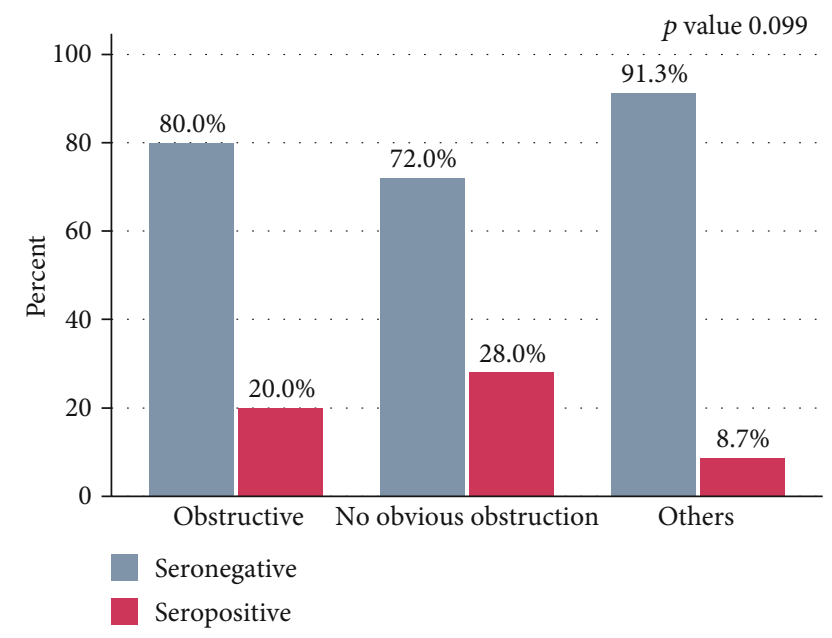

FIgURE 3: Cranial ultrasound patterns in seropositive and seronegative infants with hydrocephalus.

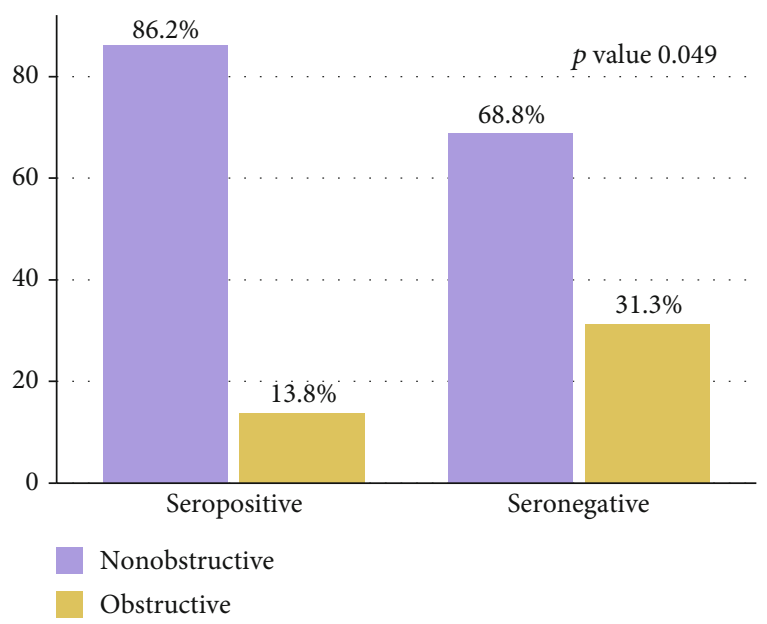

FIGURE 4: IgG seropositivity and cranial ultrasound patterns in infants with hydrocephalus. during pregnancy signifies an acute infection in the mother but not necessarily in the infant. During an acute infection, the predominant immunoglobulins are IgM, which do not cross the placenta. Of note, different studies have shown that more than half of the mothers could not recall their symptoms [37] and it is likely that these symptoms were nonspecific. Furthermore, this study did not capture information regarding treatment history of mothers to check the possibility of using some antimicrobials that can prevent transmission to fetus. However, in the study setting, there is no routine programme among pregnant women of screening for $T$. gondii and providing preventive treatment. Further studies to establish the relationship between the occurrence of $T$. gondii symptoms and congenital toxoplasmosis are warranted in this area.

In the current study, nonobstructive hydrocephalus was the most common pattern observed among infants who were IgG seropositive. This is consistent with studies in murine models which suggested that nonobstructive patterns can occur due to intraventricular or leptomeningeal inflammation, hindering cerebrospinal fluid reabsorption [38]. These findings are inconsistent though with a previous study which reported obstructive hydrocephalus to be a common feature among infants with congenital toxoplasmosis [39]. The differences could be attributed to the presence of severe hydrocephalus, the most common finding reported in the current study, which makes the assessment of congenital toxoplasma lesions by brain ultrasounds difficult as compared to brain computed tomography (brain CT scan) [17]. Further studies involving brain CT scan are warranted in this setting.

\section{Study Limitation}

A major limitation of this study was the inability to follow-up the trend in IgG antibody titers for at least two months which could confirm congenital toxoplasmosis. In addition, recall bias in obtaining retrospective information from the participants cannot be ruled out.

\section{Conclusion and Recommendations}

A significant proportion of infants with hydrocephalus have specific T. gondii IgG antibodies which is predicted by male gender, increase in birth order, and using other methods of cooking meat than boiling. Nonobstructive hydrocephalus patterns were commonly reported among infants who were T. gondii IgG seropositive. Emphasis should be put into the education of pregnant women regarding $T$. gondii risk factors, such as food preparation. In addition, there is a need to introduce serologic screening of T. gondii during pregnancy to identify infected women so that appropriate counselling and management can be provided. Moreover, routine fetal ultrasounds during pregnancy and head circumference measurements during Reproductive and Child Health $(\mathrm{RCH})$ clinic visits should be performed for those infants who are at high risk of acquiring the infection, in order to detect hydrocephalus as early as possible. 
TABLE 6: Cranial ultrasound patterns among T. gondii IgG-seropositive infants.

\begin{tabular}{|c|c|c|c|c|c|c|}
\hline $\begin{array}{l}\text { Age } \\
\text { (months) }\end{array}$ & Gender & Residence & $\begin{array}{l}\text { Maternal } \\
\text { age }\end{array}$ & Birth order & IgG titers & Brain USS \\
\hline 2 & Male & Rural & 18 & 1 & 1.94492 & Severe communicating HDC \\
\hline 1 & Male & Rural & 40 & 7 & 5.13559 & Moderate communicating HDC \\
\hline 1 & Male & Rural & 30 & 5 & 3.66525 & Mild communicating HDC \\
\hline 9 & Female & Rural & 40 & 10 & 2.52119 & Dandy Walker cysts \\
\hline 3 & Male & Rural & 17 & 1 & 1.53814 & Severe communicating HDC \\
\hline 10 & Male & Urban & 31 & 2 & 7.40254 & Lateral ventricle dilatation \\
\hline 4 & Male & Rural & 33 & 7 & 1.26871 & Paraventricular hydrocephalus post infection \\
\hline 4 & Female & Urban & 26 & 1 & 9.11905 & Severe communicating HDC \\
\hline 2 & Female & Urban & 30 & 5 & 2.44898 & Mild communicating HDC \\
\hline 2 & Male & Urban & 25 & 4 & 1.4932 & Severe communicating HDC \\
\hline 2 & Male & Urban & 35 & 1 & 1.62585 & Lateral ventricle dilatation $>>$ right side with epidural hematoma \\
\hline 1 & Male & Urban & 20 & 1 & 1.30952 & Mild communicating HDC \\
\hline 2 & Female & Rural & 24 & 3 & 2.2381 & Severe communicating HDC \\
\hline 2 & Female & Urban & 18 & 1 & 6.26531 & Severe communicating HDC \\
\hline 1 & Female & Urban & 35 & 7 & 3.29932 & Severe communicating HDC \\
\hline 9 & Male & Rural & 28 & 5 & 7.61225 & Moderate communicating HDC \\
\hline 1 & Male & Rural & 20 & 1 & 3.07483 & Severe communicating HDC \\
\hline 2 & Male & Urban & 18 & 1 & 4.49838 & Severe communicating HDC \\
\hline 3 & Male & Rural & 16 & 1 & 2.26537 & Dandy Walker cyst \\
\hline 1 & Female & Rural & 25 & 3 & 11.0356 & Severe communicating HDC \\
\hline 10 & Male & Urban & 27 & 1 & 7.83495 & Severe communicating HDC \\
\hline 1 & Female & Rural & 33 & 7 & 10.8641 & Lateral ventricle dilatation \\
\hline 1 & Female & Rural & 35 & 4 & 5.22654 & Severe communicating HDC \\
\hline 5 & Male & Rural & 38 & 6 & 2.884 & Moderate communicating HDC \\
\hline 7 & Male & Urban & 30 & 5 & 1.656 & Mild communicating HDC \\
\hline 12 & Male & Rural & 39 & 9 & 1.252 & Severe communicating HDC \\
\hline 7 & Male & Urban & 31 & 2 & 3.408 & Severe communicating HDC \\
\hline 11 & Male & Rural & 32 & 7 & 3.12 & Lateral ventricle dilatation \\
\hline 11 & Male & Rural & 29 & 5 & 3.432 & Severe communicating HDC \\
\hline
\end{tabular}

\section{Data Availability}

All data collected have been used for the current article. All data are available on request to the Director of Research \& Innovations.

\section{Ethical Approval}

Ethical clearance for the study was obtained from the joint CUHAS/BMC Research Ethics and Review Committee with the ethical clearance number CREC/210/2017.

\section{Consent}

Prior to recruitment, the purpose of the study was explained to the participants. Information sheets were provided to participants' parents/guardians in a Swahili version to ensure the understanding of the participants. Participants' parents or guardians were required to sign consent forms prior to the involvement of their children in the study. Confidentiality was ensured and findings relevant to the child's management were shared with clinicians who were attending the children.

\section{Conflicts of Interest}

The authors declare that they have no competing interests.

\section{Authors' Contributions}

DM, MMM, RK, SO, and SEM participated in designing the study. SO, MS, RK, PN, and DRM participated in data/sample collection, clinical evaluation, and management of the children; MMM and SEM carried out the laboratory procedures; BRK and SEM participated in data analysis; MMM, $\mathrm{RK}, \mathrm{SO}$, and SEM interpreted the data. MMM wrote the first draft of the manuscript. SEM and DM did the critical review of the manuscript. All authors approved the last version of the manuscript. 


\section{Acknowledgments}

The authors would like to express their sincere gratitude to the Catholic University of Health and Allied Sciences and the Department of Radiology, Bugando Medical Centre, for supporting this study. The funding for this research has been provided by the Catholic University of Health and Allied Sciences and the Bugando Medical Centre from their respective research funds for training.

\section{References}

[1] V. N. Klaren and A. Kijlstra, "Toxoplasmosis, an overview with emphasis on ocular involvement," Ocular Immunology and Inflammation, vol. 10, no. 1, pp. 1-26, 2009.

[2] D. Dunn, M. Wallon, F. Peyron, E. Petersen, C. Peckham, and R. Gilbert, "Mother-to-child transmission of toxoplasmosis: risk estimates for clinical counselling," The Lancet, vol. 353, no. 9167, pp. 1829-1833, 1999.

[3] J. Negero, M. Yohannes, K. Woldemichael, and D. Tegegne, "Seroprevalence and potential risk factors of T. gondii infection in pregnant women attending antenatal care at Bonga Hospital, Southwestern Ethiopia," International Journal of Infectious Diseases, vol. 57, pp. 44-49, 2017.

[4] B. Mwambe, S. E. Mshana, B. R. Kidenya et al., "Sero-prevalence and factors associated with Toxoplasma gondii infection among pregnant women attending antenatal care in Mwanza, Tanzania," Parasites \& Vectors, vol. 6, no. 1, p. 222, 2013.

[5] M. N. Spann, A. Sourander, H. M. Surcel, S. Hinkka-Yli-Salomäki, and A. S. Brown, "Prenatal toxoplasmosis antibody and childhood autism," Autism Research, vol. 10, no. 5, pp. 769777, 2017.

[6] H. L. Rekate, A contemporary definition and classification of hydrocephalus, Elsevier, 2009.

[7] T. D. Simon, J. Riva-Cambrin, R. Srivastava et al., "Hospital care for children with hydrocephalus in the United States: utilization, charges, comorbidities, and deaths," Journal of Neurosurgery: Pediatrics, vol. 1, no. 2, pp. 131-137, 2008.

[8] J. H. Chi, H. J. Fullerton, and N. Gupta, “Time trends and demographics of deaths from congenital hydrocephalus in children in the United States: National Center for Health Statistics data, 1979 to 1998," Journal of Neurosurgery: Pediatrics, vol. 103, 2 Suppl, pp. 113-118, 2005.

[9] B. C. Warf, "Pediatric hydrocephalus in East Africa: prevalence, causes, treatments, and strategies for the future," World Neurosurgery, vol. 73, no. 4, pp. 296-300, 2010.

[10] T. R. Olariu, J. S. Remington, R. McLeod, A. Alam, and J. G. Montoya, "Severe congenital toxoplasmosis in the United States: clinical and serologic findings in untreated infants," The Pediatric Infectious Disease Journal, vol. 30, no. 12, pp. 1056-1061, 2011.

[11] F. Mashuda, A. Zuechner, P. L. Chalya, B. R. Kidenya, and M. Manyama, "Pattern and factors associated with congenital anomalies among young infants admitted at Bugando medical centre, Mwanza, Tanzania," BMC Research Notes, vol. 7, no. 1, p. 195, 2014.

[12] H. M. Seitz, N. A. S. Hamudu, H. Hlobil et al., "Toxoplasma gondii antibodies in pregnant women and their newborns in Dar es Salaam, Tanzania," The American Journal of Tropical Medicine and Hygiene, vol. 52, no. 6, pp. 546-548, 1995.
[13] I. Machumi, M. M Mirambo, D. Ruganuza et al., "Factors associated with Toxoplasma gondii $\operatorname{IgG}$ and $\operatorname{IgM}$ antibodies, and placental histopathological changes among women with spontaneous abortion in Mwanza City, Tanzania," East African Health Research Journal, vol. 1, no. 2, pp. 86-94, 2017.

[14] Health Mo, Standard Treatment Guidlines and Essential Medicne List and Laboratory Supplies List, Ministry of Health, Zambia, 2008.

[15] M. Paul, E. Petersen, Z. S. Pawlowski, and J. Szczapa, "Neonatal screening for congenital toxoplasmosis in the Poznan region of Poland by analysis of Toxoplasma gondii-specific IgM antibodies eluted from filter paper blood spots," The Pediatric Infectious Disease Journal, vol. 19, no. 1, pp. 3036, 2000.

[16] N. Zainodini, M. Zare-Bidaki, S. H. Abdollahi et al., "Molecular and serological detection of acute and latent toxoplasmosis using real-time PCR and ELISA techniques in blood donors of Rafsanjan City, Iran, 2013," Iranian Journal of Parasitology, vol. 9, no. 3, pp. 336-341, 2014.

[17] S. L. Hutson, K. M. Wheeler, D. McLone et al., "Patterns of hydrocephalus caused by congenital Toxoplasma gondii infection associate with parasite genetics," Clinical Infectious Diseases, vol. 61, no. 12, pp. 1831-1834, 2015.

[18] J. M. Hughes, D. G. Colley, A. Lopez et al., Preventing congenital toxoplasmosis, Morbidity and Mortality Weekly Report: Recommendations and Reports, Centers for Disease Control \& Prevention (CDC), 2000.

[19] D. V. Vasconcelos-Santos, D. O. Machado Azevedo, W. R. Campos et al., "Congenital toxoplasmosis in southeastern Brazil: results of early ophthalmologic examination of a large cohort of neonates," Ophthalmology, vol. 116, no. 11, pp. 2199-2205.e1, 2009.

[20] J. S. Remington and J. O. Klein, Infectious Diseases of the Fetus and Newborn Infant, WB Saunders Co, 1983.

[21] M. M. Mirambo, C. Kivambe, M. F. Mushi et al., "High seroprevalence of specific Toxoplasma gondii IgG antibodies among HIV/AIDS patients with immunological failure attending a tertiary hospital in northwestern Tanzania," Tanzania Journal of Health Research, vol. 18, no. 1, 2016.

[22] D. J. Ferguson, “Toxoplasma gondii: 1908-2008, homage to Nicolle, Manceaux and Splendore," Memorias do Instituto Oswaldo Cruz, vol. 104, no. 2, pp. 133-148, 2009.

[23] N. G. Guerina, H.-W. Hsu, H. C. Meissner et al., "Neonatal serologic screening and early treatment for congenital Toxoplasma gondii infection," New England Journal of Medicine, vol. 330, no. 26, pp. 1858-1863, 1994.

[24] Y. Suzuki and A. Kobayashi, "Induction of tolerance to Toxoplasma gondii in newborn mice by maternal antibody," Parasitology Research, vol. 76, no. 5, pp. 424-427, 1990.

[25] M. A. Abu-Madi, N. Al-Molawi, and J. M. Behnke, "Seroprevalence and epidemiological correlates of Toxoplasma gondii infections among patients referred for hospital-based serological testing in Doha, Qatar," Parasites \& Vectors, vol. 1, no. 1, p. 39, 2008.

[26] J. Montoya and O. Liesenfeld, "Toxoplasmosis," Lancet, vol. 363, no. 9425, pp. 1965-1976, 2004.

[27] E. Zemene, D. Yewhalaw, S. Abera, T. Belay, A. Samuel, and A. Zeynudin, "Seroprevalence of Toxoplasma gondiiand associated risk factors among pregnant women in Jimma town, southwestern Ethiopia," BMC Infectious Diseases, vol. 12, no. $1,2012$. 
[28] J. L. Sanders, Y. Zhou, Z. Moulton et al., "The zebrafish, Danio rerio, as a model for Toxoplasma gondii: an initial description of infection in fish," Journal of Fish Diseases, vol. 38, no. 7, pp. 675-679, 2015.

[29] K. Shapiro, L. Bahia-Oliveira, B. Dixon et al., "Environmental transmission of Toxoplasma gondii: oocysts in water, soil and food," Food and Waterborne Parasitology, vol. 15, article e00049, 2019.

[30] J. Dubey and J. Jones, "Toxoplasma gondii infection in humans and animals in the United States," International Journal for Parasitology, vol. 38, no. 11, pp. 1257-1278, 2008.

[31] J. Dubey, "Toxoplasmosis in sheep-the last 20 years," Veterinary Parasitology, vol. 163, no. 1-2, pp. 1-14, 2009.

[32] J. P. Dubey, Toxoplasmosis of animals and humans, CRC press, 2016.

[33] P. Santos, G. Albuquerque, V. M. F. da Silva et al., "Seroprevalence of Toxoplasma gondii in free-living Amazon River dolphins (Inia geoffrensis) from Central Amazon, Brazil," Veterinary Parasitology, vol. 183, no. 1-2, pp. 171-173, 2011.

[34] P. Conrad, M. Miller, C. Kreuder et al., "Transmission of Toxoplasma: clues from the study of sea otters as sentinels of Toxoplasma gondii flow into the marine environment," International Journal for Parasitology, vol. 35, no. 11-12, pp. 1155-1168, 2005.

[35] M. Koskiniemi, M. Lappalainen, and K. Hedman, "Toxoplasmosis needs evaluation: an overview and proposals," American Journal of Diseases of Children, vol. 143, no. 6, pp. 724-728, 1989.

[36] J. Dubey, "Sources of Toxoplasma gondii infection in pregnancy: until rates of congenital toxoplasmosis fall, control measures are essential," BMJ: British Medical Journal, vol. 321, no. 7254, pp. 127-128, 2000.

[37] K. M. Boyer, E. Holfels, N. Roizen et al., "Risk factors for Toxoplasma gondii infection in mothers of infants with congenital toxoplasmosis: implications for prenatal management and screening," American Journal of Obstetrics \& Gynecology, vol. 192, no. 2, pp. 564-571, 2005.

[38] A. V. Ciurea, T. C. Coman, and D. Mircea, Postinfectious hydrocephalus in children, Springer, Pediatric Hydrocephalus, 2005.

[39] M. Jacob, B. E. Kasilima, and R. Kazema, "Investigation of infants with hydrocephalous at Muhimbili National Hospital using anterior transfontanelle ultrasound scan," Tanzania Medical Journal, vol. 27, no. 1, 2015. 